\section{Fortbildungszentrum in neuem Glanz wiedereröffnet}

\section{Die Neueröffnung des firmeneigenen Fortbildungszentrums am Standort in Ellwangen fand in Anwesenheit geladener \\ Gäste statt.}

Der dentalen Fachwelt steht nunmehr eines der modernsten industriellen Fortbildungszentren ihrer Branche zur Verfügung. Das ICDE (International Center for Dental Education) gilt schon seit Jahren als Institution. Mit hohem Aufwand modernisiert, erstrahlt es jetzt in neuem Glanz. Ausgestattet mit modernster Präsentations- und Anwendungstechnik, muss das ICDE keinen Vergleich scheuen. Auf $750 \mathrm{~m}^{2}$ befinden sich unter anderem ein Hörsaal mit 72 Plätzen und Multimediatechnik sowie 3 zahntechnische Labore mit insgesamt 30 Arbeitsplätzen. Neben allen konventionellen Techniken verfügt das ICDE nun insbesondere auch über eine Komplettausstattung für die CAD/ CAM-basierte digitale Zahnmedizin und Zahntechnik.

\section{Trend zur Digitalisierung}

Die Digitalisierung in der Dentalbranche hat mächtig Fahrt aufgenommen. Sie verändert die Arbeitsprozesse in der Zahnarztpraxis und im Dentallabor. „Dieser Entwicklung tragen wir Rechnung“, erklärt Norbert Wild, Geschäftsführer der Ivoclar Vivadent GmbH. „Unser ICDE verfügt über ideale Voraussetzungen, um praxisrelevantes Wissen und moderne Anwendungstechnologie zu vermitteln. So wollen wir mit dazu beitragen, künftige Herausforderungen in dieser Branche aktiv zu gestalten.“

\section{Seit Jahren erfolgreich}

Das ICDE blickt auf eine Erfolgsgeschichte zurück. Mehr als 70000 Besucher aus der Dentalbranche haben sich seit der ersten Eröffnung 1987 dort weitergebildet. Dar-

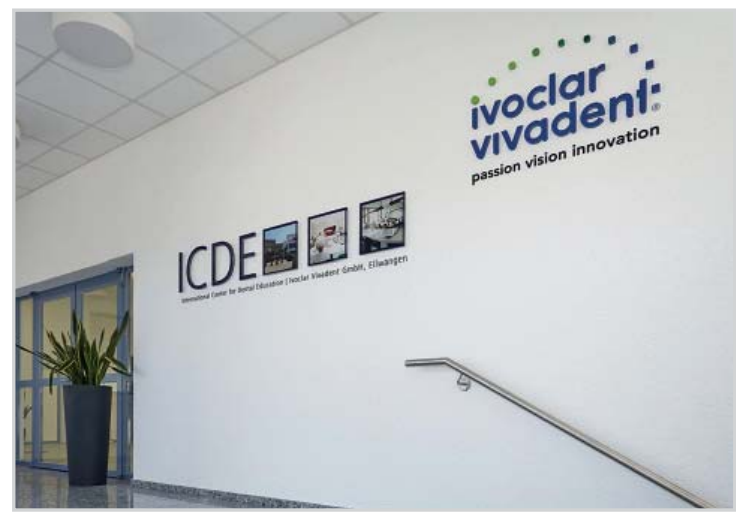

Der Eingangsbereich zum neu gestalteten ICDE

über hinaus ist Ivoclar Vivadent bundesweit in der dentalen Fortbildung aktiv. Referenten aus Praxis, Labor und Hochschule vermitteln in Symposien und Seminaren fundiertes Expertenwissen und bieten Gelegenheit zum Austausch. Mehr als 19000 Zahnärzte und Zahntechniker nahmen allein in den letzten 5 Jahren an solchen Veranstaltungen teil. „Die Basis unseres Erfolges sind hochkarätige Referenten, tolle Veranstaltungsorte und HD-Präsentationstechnik“, erklärt der Verantwortliche für das Fortbildungsprogramm, Dr. Frank Drubel.

Nach einer Pressemitteilung der Ivoclar Vivadent GmbH, Ellwangen Internet: www.ivoclarvivadent.de 